\title{
Atividade de enzimas do sistema de defesa antioxidante de tilápias (Oreochromis niloticus) como modelo de exposição à nanopartícula de dióxido de titânio $\left(\mathrm{TiO}_{2}\right)$
}

\author{
Enzyme activity of the tilapia (Oreochromis niloticus) antioxidant defense system as a model of \\ exposure to the titanium dioxide $\left(\mathrm{TiO}_{2}\right)$ nanoparticle \\ Actividad enzimática del sistema de defensa antioxidante de la tilapia (Oreochromis niloticus) como \\ modelo de exposición a la nanopartícula de dióxido de titanio $\left(\mathrm{TiO}_{2}\right)$
}

Recebido: 10/02/2021 | Revisado: 20/04/2021 |Aceito: 29/04/2021 | Publicado: 13/05/2021

Theila dos Santos Santana
ORCID: https://orcid.org/0000-0001-7093-2565
Universidade Federal do Recôncavo da Bahia, Brasil
E-mail: theilaribeirosantana@gmail.com
Marilene Bárbara dos Santos
ORCID: https://orcid.org/0000-0002-9082-5780
Universidade Federal do Recôncavo da Bahia, Brasil
E-mail: marilenebarbara_mbs@ @otmail.com
Elissandra Ulbricht Winkaler
ORCID: https://orcid.org/0000-0003-3297-4283
Universidade Federal do Recôncavo da Bahia, Brasil
E-mail: elis@ ufrb.edu.br

\begin{abstract}
Resumo
Os biomarcadores bioquímicos de peixes podem ser ferramentas úteis na investigação da presença de nanopartículas (NPs) no sistema aquático. Entretanto, é de suma importância conhecer as respostas desses biomarcadores frente a exposição a esse xenobiotico para se tentar estabelecer um modelo para monitoramento biológico. Assim, nosso objetivo foi determinar a resposta das enzimas catalase (CAT) e glutationa S-transferase (GST) de tilápias (Oreochromis niloticus) após 48 e 96h de exposição a nanopartícula de dióxido de titânio (NP- $\mathrm{TiO}_{2}$ ). Para tanto, 10 peixes foram expostos a: 0 (controle), 1, 5, 10 e $50 \mathrm{mg} \cdot \mathrm{L}^{-1}$ da $\mathrm{NP}^{-\mathrm{TiO}_{2}}$. Em seguida, o fígado foi utilizado para a determinação dos biomarcadores. Nós não observamos variação significativa da CAT nas tilápias expostas durante 48h a NP. Entretanto, após 96h a CAT foi maior nos animais expostos as maiores concentrações testadas (10 e 50 mg. $\mathrm{L}^{-1}$ ). Ainda nessas concentrações, a atividade foi maior nos animais expostos durante $96 \mathrm{~h}$. Para a GST, nas primeiras $48 \mathrm{~h}$ a atividade foi menor nos animais expostos a 1,5 e $50 \mathrm{mg} \cdot \mathrm{L}^{-1} \mathrm{de} \mathrm{TiO}_{2}$, enquanto que, na exposição de $96 \mathrm{~h}$ a maior atividade da GST foi observada nos animais expostos a $50 \mathrm{mg} . \mathrm{L}^{-1}$. Comparando-se o tempo de exposição, em todas as concentrações testadas, a atividade da GST hepática foi maior nos animais expostos durante 96h. Assim, além da concentração, o tempo de exposição a NP parece influenciar na resposta das enzimas, aumentando sua atividade.
\end{abstract}

Palavras-chave: Nanoecotoxicologia; Enzimas antioxidantes; Catalase; Glutationa S-transferase.

\begin{abstract}
The biochemical biomarkers of fish can be useful tools in the investigation of the presence of nanoparticles (NPs) in the aquatic system. However, it is extremely important to know the responses of these biomarkers to exposure to this xenobiotic in order to try to establish a model for biological monitoring. Thus, our objective was to determine the response of the enzymes catalase (CAT) and glutathione S-transferase (GST) of tilapia (Oreochromis niloticus) after 48 and 96 hours of exposure to a titanium dioxide nanoparticle $\left(\mathrm{NP}^{\left.-\mathrm{TiO}_{2}\right)}\right)$. For this purpose, 10 fish were exposed to:

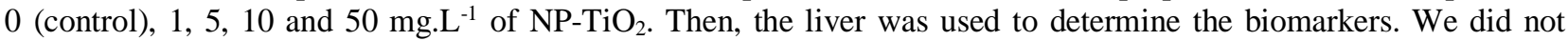
observe a significant variation in CAT in tilapia exposed for $48 \mathrm{~h}$ to PN. However, after $96 \mathrm{~h}$ the CAT was higher in the exposed animals at the highest tested concentrations $\left(10\right.$ and $\left.50 \mathrm{mg} . \mathrm{L}^{-1}\right)$. Even at these concentrations, the activity was higher in the animals exposed for 96 hours. For GST, in the first $48 \mathrm{~h}$ the activity was lower in animals exposed to 1,5 and $50 \mathrm{mg} \cdot \mathrm{L}^{-1}$ of $\mathrm{TiO}_{2}$, whereas, in the exposure of $96 \mathrm{~h}$, greater activity of GST was observed in animals exposed to $50 \mathrm{mg} . \mathrm{L}^{-1}$. Comparing the exposure time, in all concentrations tested, the hepatic GST activity was higher in the animals exposed for $96 \mathrm{~h}$. Thus, in addition to the concentration, the time of exposure to NP seems to influence the response of the enzymes, increasing their activity.
\end{abstract}

Keywords: Nanoecotoxicology; Antioxidant enzymes; Catalase; Glutathione S-transferase. 


\begin{abstract}
Resumen
Los biomarcadores bioquímicos de los peces pueden ser herramientas útiles en la investigación de la presencia de nanopartículas (NP) en el sistema acuático. Sin embargo, es de suma importancia conocer las respuestas de estos biomarcadores a la exposición a este xenobiótico para intentar establecer un modelo de seguimiento biológico. Así, nuestro objetivo fue determinar la respuesta de las enzimas catalase (CAT) y glutatión S-transferase (GST) de tilapia (Oreochromis niloticus) tras 48 y 96 horas de exposición a una nanopartícula de dióxido de titanio $\left(\mathrm{NP}-\mathrm{TiO}_{2}\right)$. Para ello, se expusieron 10 peces a: 0 (control), $1,5,10$ y $50 \mathrm{mg} \cdot \mathrm{L}^{-1}$ de $\mathrm{NP}-\mathrm{TiO}_{2}$. Luego, se utilizó el hígado para determinar los biomarcadores. No observamos una variación significativa en CAT en tilapia expuesta durante $48 \mathrm{~h}$ a NP. Sin embargo, después de 96 h, el CAT fue mayor en los animales expuestos a las concentraciones más altas probadas (10 y $50 \mathrm{mg} . \mathrm{L}^{-1}$ ). Incluso a estas concentraciones, la actividad fue mayor en los animales expuestos durante $96 \mathrm{~h}$. Para GST, en las primeras $48 \mathrm{~h}$ la actividad fue menor en animales expuestos a 1,5 y $50 \mathrm{mg} . \mathrm{L}^{-1}$ de $\mathrm{TiO}_{2}$, mientras que, en la exposición de $96 \mathrm{~h}$, se observó mayor actividad de GST en animales expuestos a $50 \mathrm{mg} . \mathrm{L}^{-1}$. Comparando el tiempo de exposición, en todas las concentraciones probadas, la actividad de GST hepática fue mayor en los animales expuestos durante 96 h. Así, además de la concentración, el tiempo de exposición a NP parece influir en la respuesta de las enzimas, aumentando su actividad.
\end{abstract}

Palabras clave: Nanoecotoxicología; Enzimas antioxidantes; Catalase; Glutatión S-transferase.

\title{
1. Introdução
}

A nanotecnologia inclui a aplicação de material com nanoescala, que possuem dimensões na faixa de 1 a $100 \mathrm{~nm}$. Dentre essas, destaca-se a nanaopartícula (NP) de dióxido de titânio $\left(\mathrm{TiO}_{2}\right)$, com produção anual acima de 10.000 toneladas e com previsão de atingir 2,5 milhões de toneladas até 2025 (Mahaling \& Dinabandhu, 2018). As $\mathrm{NPs}^{-\mathrm{TiO}_{2}}$ são comumente usadas em produtos industriais, incluindo tintas, materiais têxteis, papéis, plásticos, protetores solares, cosméticos, sensores eletrônicos e também utilizados na indústria de alimentos (Martirosyan \& Schneider, 2014).

Devido a grande utilização das NPs, a estimativa é que entre 63 e $91 \%$ do total de toneladas de nanopartículas manufaturadas produzidas acabaram em aterros ou foram liberados no solo e na atmosfera. Desse montante, até $7 \%$ podem ter alcançado os corpos de água (Keller, McFerran, Lazareva \& Suh, 2013). Os organismos aquáticos são mais suscetíveis a contaminação do ambiente e podem ser considerados bons bioindicadores (Pirsaheb et al., 2019). Em vários trabalhos foi possível evidenciar o potencial tóxico de $\mathrm{NPs}^{-\mathrm{TiO}_{2}}$ em peixes (Karthigarani \& Navaraj, 2012; Varela-Valencia et al., 2014; Hajirezaeea et al., 2020) devido ao aumento na produção de Espécies Reativas de Oxigênio (EROs) (Reeves, Davies, Dodd \& Jha, 2008; Hao, Wang \& Xing, 2009; Carmo et al., 2018; Carmo et al., 2019).

Todos os organismos, incluindo os peixes, possuem um sistema de defesa antioxidante formado por enzimas responsáveis em neutralizar as EROs produzidos em excesso (Paital, 2018). Assim, a análise da atividade dessas enzimas pode indicar o status antioxidante dos organismos, funcionando como um potencial biomarcador bioquímico para o estresse oxidativo (Valavanidis, Vlahogianni, Dassenakis \& Scoullos, 2006) induzido pela presença de NPs na água.

Em estudos nanotoxicológicos, os biomarcadores podem ser úteis, pois são indicadores chave de alerta precoce dos riscos para os seres humanos e para os ecossistemas (Pham \& Gu, 2012). Segundo Clemente et al. (2013), a exposição de organismos aquáticos a NP-TiO 2 pode induzir o aumento ou decréscimo na atividade de enzimas antioxidantes como a catalase (CAT), superóxido dismutase, glutationa S-transferase (GST) e a peroxidase. A enzima CAT está presente em organismos aeróbicos e anaeróbicos e sua função é diminuir a toxicidade do peróxido de hidrogênio. A GST, além da desintoxicação, atua como nos processos de biotransformação, tornando o xenobiótico menos tóxico para a célula (Benavides, Fernandez-Lodeiro, Coelho, Lodeiro \& Diniz, 2016). Ambas as enzimas estão envolvidas no processo de defesa contra as EROs e suas respostas têm sido empregadas para avaliar os efeitos da exposição a nanomateriais no nível celular.

Tendo em vista o atual cenário de expansão do uso de $\mathrm{NPs}^{-\mathrm{TiO}_{2}}$ e o seu potencial tóxico para os organismos aquáticos, o presente estudo tem como objetivo analisar o efeito da exposição aguda (48 e 96 h) a diferentes concentrações de NP-TiO 2 em peixes (Oreochromis niloticus Linnaeus, 1758), utilizando a atividade das enzimas CAT e GST como biomarcadores bioquímicos. 


\section{Metodologia}

\subsection{Bioindicadores e preparo das soluções de $\mathrm{NP}^{-\mathrm{TiO}_{2}}$}

A realização do experimento foi aprovado pelo Comitê de Ética Animal da Universidade Federal do Recôncavo da Bahia (Protocolo n 23007. 001517/2016-27). Para tanto, exemplares jovens de tilápias, Oreochromis niloticus, fornecidos pela piscicultura Aqua Vale (Ituberá, BA) foram transferidos para o Laboratório de Ecotoxicologia Aquática (LABEA) da Universidade Federal do Recôncavo da Bahia (UFRB).

Os animais foram aclimatados por 60 dias em tanques de polietileno $(250 \mathrm{~L})$, aeração contínua e fotoperíodo controlado (12Claro/12Escuro). A água do tanque foi renovada diariamente e os peixes alimentados ad libitum. As variáveis físico-químicas da água (temperatura: $24,4 \pm 0,3{ }^{\circ} \mathrm{C}$, oxigênio dissolvido: $6,8 \pm 0,5 \mathrm{mg} . \mathrm{L}^{-1}$, pH: $6,8 \pm 0,4$, condutividade: $410,3 \pm 50,1 \mu \mathrm{S} \mathrm{cm}^{-3}$ e salinidade: $0,2 \pm 0,1 \%$ ) foram monitoradas diariamente e se mantiveram constantes durante o período de aclimatação.

As concentrações da nanopartícula de $\mathrm{TiO}_{2}\left(\mathrm{NP}-\mathrm{TiO}_{2}\right)$, correspondentes a 1, 5, 10 e 50 mg.L ${ }^{-1}$ (concentração nominal), foram preparadas a partir do nano pó de $\mathrm{TiO}_{2}$ anatase (Sigma-Aldrich®), com 99,7 \% de pureza, $21 \mathrm{~nm}$ de tamanho da particula, superfície específica de $45-55 \mathrm{~m}^{2} \cdot \mathrm{g}^{-1}$ e diluídas em água ultrapura. Em seguida, as suspensões foram homogeneizadas em banho maria com ultrassom por 40 minutos a $37 \mathrm{KHz}$. As soluções foram preparadas no mesmo dia dos testes, evitando aglutinação das nanopartículas.

\subsection{Delineamento experimental}

Para a realização dos testes, 10 exemplares de $O$. niloticus foram distribuídos aleatoriamente em 10 aquários de vidro (30 L) e foram aclimatados por $24 \mathrm{~h}$. Em seguida, foram adicionados aos aquários as soluções de 0 (grupo controle), 1, 5, 10 e $50 \mathrm{mg} . \mathrm{L}^{-1}$ da $\mathrm{NP}-\mathrm{TiO}_{2}$. No grupo controle (CTR), os peixes foram expostos somente a água de abastecimento do laboratório, sem a presença da nanopartícula. Os animais não foram alimentados durante os experimentos e a oxigenação da água, garantida por sopradores de ar. O teste foi do tipo estático com fotoperíodo (12Claro/12Escuro) controlado com timer digital.

Após 48 e 96 h de exposição a NP-TiO 2 , os animais foram retirados individualmente dos aquários e anestesiados por hipotermia em caixas isotérmicas contendo gelo e água (1:1). Ainda anestesiados, os animais foram pesados e medidos (massa corpórea: $30,7 \pm 4,8 \mathrm{~g}$, comprimento total: $12,2 \pm 0,7 \mathrm{~cm}$ e comprimento padrão: 9,6 $\pm 0,7 \mathrm{~cm}$ ). Em seguida foram eutanasiados via secção medular e o fígado foi e armazenado em freezer $-80^{\circ} \mathrm{C}$, para as análises bioquímicas. Durante todo período de exposição a $\mathrm{NP}^{-} \mathrm{TiO}_{2}$, os parâmetros da água (oxigênio dissolvido, pH, temperatura, condutividade e salinidade) foram mensurados com auxilio de sonda multiparâmetro (Hanna HI9828) a cada 24 horas (Tabela 1). Os valores mantiveram-se, de modo geral, dentro dos parâmetros ideais estabelecidos pela ABNT NBR 15088, assim, nós consideramos que as variáveis não influenciaram nos resultados. 
Tabela 1. Valores dos parâmetros abióticos da água durante a realização dos testes de exposição aguda (48 e 96 h) de tilápias, Oreochromis niloticus, a diferentes concentrações da nanopartícula de dióxido de titânio $\left(\mathrm{NP}-\mathrm{TiO}_{2}\right)$.

\begin{tabular}{cccccc}
\hline $\begin{array}{c}\text { Concentração* } \\
\begin{array}{c}\text { NP-TiO } \\
\left(\mathrm{mg.L}^{-1}\right)\end{array}\end{array}$ & $\mathrm{pH}$ & $\begin{array}{c}\text { Temperatura } \\
\left({ }^{\circ} \mathrm{C}\right)\end{array}$ & $\begin{array}{c}\text { Condutividade } \\
\left.(\mathrm{mS.cm})^{-1}\right)\end{array}$ & $\begin{array}{c}\text { Salinidade } \\
(\% \mathrm{oo})\end{array}$ & $\begin{array}{c}\text { Oxigênio } \\
\text { dissolvido } \\
\left(\mathrm{mg.L}^{-1}\right)\end{array}$ \\
\hline \hline $0(\mathrm{CTR})$ & $7,2 \pm 0,3$ & $24,3 \pm 0,2$ & $387,0 \pm 29,9$ & $0,2 \pm 0,1$ & $5,1 \pm 0,4$ \\
\hline 1 & $7,0 \pm 0,4$ & $24,3 \pm 0,3$ & $375,4 \pm 20,0$ & $0,2 \pm 0,0$ & $5,4 \pm 0,7$ \\
\hline 5 & $7,0 \pm 0,3$ & $24,4 \pm 0,2$ & $415,3 \pm 81,8$ & $0,2 \pm 0,0$ & $5,9 \pm 0,1$ \\
\hline 10 & $6,9 \pm 0,3$ & $24,3 \pm 0,2$ & $542,3 \pm 26,4$ & $0,2 \pm 0,1$ & $5,9 \pm 0,1$ \\
\hline 50 & $6,9 \pm 0,5$ & $24,4 \pm 0,2$ & $438,8 \pm 25,1$ & $0,2 \pm 0,0$ & $5,3 \pm 0,7$ \\
\hline \hline
\end{tabular}

*Concentração nominal. Valores expressos como média \pm desvio padrão de cinco medidas: 0, 24, 48, 72 e 96 h após o inicio da exposição aguda. Fonte: Autores.

\subsection{Determinação dos biomarcadores: catalase e glutationa S-transferase}

Para a determinação dos biomarcadores bioquímicos, as amostras de fígado foram pesadas e homogeneizadas na proporção de 1:10 (m/v) em tampão fosfato de potássio (Tris-HCl, $50 \mathrm{mM}, 0,15 \mathrm{M} \mathrm{KCl,} \mathrm{pH} \mathrm{6,8).} \mathrm{O} \mathrm{homogeneizado} \mathrm{do} \mathrm{foi}$ centrifugado durante 25 minutos $\left(4^{\circ} \mathrm{C}\right)$ a $12.300 \mathrm{~g}$. Após esse procedimento, o sobrenadante foi separado e congelado a $-80^{\circ} \mathrm{C}$, para posterior determinação da atividade das enzimas CAT e GST.

A atividade da CAT ( $\mu \mathrm{mol} \mathrm{H}_{2} \mathrm{O}_{2} \mathrm{~min}^{-1} \mathrm{mg} \cdot \mathrm{pt}^{-1}$ ) foi determinada com base na degradação do $\mathrm{H}_{2} \mathrm{O}_{2}$ exógeno pela CAT, gerando como subproduto a $\mathrm{H}_{2} \mathrm{O}$ e $\mathrm{O}_{2}$ (Aebi, 1984). As leituras foram realizadas no espectrofotômetro UV/Vis (software Reaction kinetics) em cubetas de quartzo e comprimento de $240 \mathrm{~nm}$. A atividade da GST (nmol CDNB min ${ }^{-1} \mathrm{mg} \mathrm{pt}^{-1}$ ) foi determinada com base na catalisação da reação de conjugação do substrato 1-26 cloro-2,4-dinitrobenzeno (CDNB) com a glutationa reduzida, no comprimento de $340 \mathrm{~nm}$ em cubetas de acrílico (Keen, Habig \& Jakoby, 1976). A concentração de proteína total hepática foi mensurada utilizando-se kit comercial (Interkit@).

\subsection{Análise estatística}

A atividade da CAT e GST foi comparada estatisticamente pelo teste paramétrico ANOVA two way, onde foram utilizadas duas variáveis: tempo de exposição e concentrações de $\mathrm{NP}^{-} \mathrm{TiO}_{2}$ com posterior teste de Tukey. As análises foram realizadas com o auxílio do programa Past versão 2.17 C. Na ocorrência de variações entre as médias $(\mathrm{p}<0,05)$ o teste de TUKEY foi aplicado para identificar os grupos que se distinguem.

\section{Resultados e Discussão}

$\mathrm{O}$ mecanismo de toxicidade da $\mathrm{NP}-\mathrm{TiO}_{2}$ para os organismos pode ser descrito em três aspectos: (1) Produção de Espécies Reativas de Oxigênio (EROs) após a indução de pares radicais livres na presença de irradiação UV; (2) Fluxo de citoplasma e ligação da $\mathrm{NP}-\mathrm{TiO}_{2}$ a organelas intracelulares e macromoléculas biológicas, após danos às membranas celulares e; (3) Danos na parede celular e peroxidação lipídica da membrana celular causada pela fixação de células da NP por força eletrostática, devido à grande área superficial (Hou et al., 2019).

Esse dano oxidativo, devido à presença das NPs, pode ser neutralizado pelo sistema de defesa antioxidante e por mecanismos de reparo, que inclui enzimas que regulam os intermediários reativos produzidos nas células (Paital, 2018). A enzima superóxido dismutase catalisa a dismutação do radical superóxido $\left(\mathrm{O}_{2}{ }^{-}\right)$em $\mathrm{O}_{2}$ e $\mathrm{H}_{2} \mathrm{O}_{2}$ enquanto que, a catalase (CAT) catalisa a conversão de $\mathrm{H}_{2} \mathrm{O}_{2}$, que é tóxico para as células em $\mathrm{H}_{2} \mathrm{O}$ e $\mathrm{O}_{2}$ (Benavides et al., 2016). 
Em nosso trabalho, não observamos variação significativa $(\mathrm{F}=1,04$ e $\mathrm{p}=0,45)$ na atividade da CAT no fígado das tilápias expostas durante $48 \mathrm{~h}$ as diferentes concentrações da nanopartícula de $\mathrm{TiO}_{2}$. A atividade da CAT também não variou em relação ao grupo controle (CTR), onde os animais foram expostos somente a água de diluição (Figura 1). Entretanto, após 96 horas de exposição, a atividade da CAT foi significativamente maior $(\mathrm{F}=13,03$ e $\mathrm{p}=0,00)$ nos animais expostos as concentrações as maiores concentrações $\left(10\right.$ e $\left.50 \mathrm{mg} \cdot \mathrm{L}^{-1}\right)$ da $\mathrm{NP}^{-\mathrm{TiO}_{2}}$, quando comparada com o grupo CTR e as concentrações de 1 e $5 \mathrm{mg} . \mathrm{L}^{-1}$ de $\mathrm{TiO}_{2}$.

Figura 1. Atividade da catalase (CAT) hepática de tilápia (O. Niloticus) expostas a 0 (controle), 1, 5,10 e 50 mg.. $\mathrm{L}^{-1}$ da nanopartícula de $\mathrm{TiO}_{2}$ após 48 e 96 horas de exposição. Valores apresentados como média e desvio padrão. Letras iguais indicam valores sem diferença significativa $(p>0,05)$ e letras diferentes indicam valores estatisticamente diferentes $(p<0,05)$. Letras maiúsculas referentes à diferença entre as concentrações, letras minúsculas diferença referente ao tempo de exposição dentro do mesmo grupo. $\left(^{*}\right)$ indica diferença significativa entre o grupo controle.

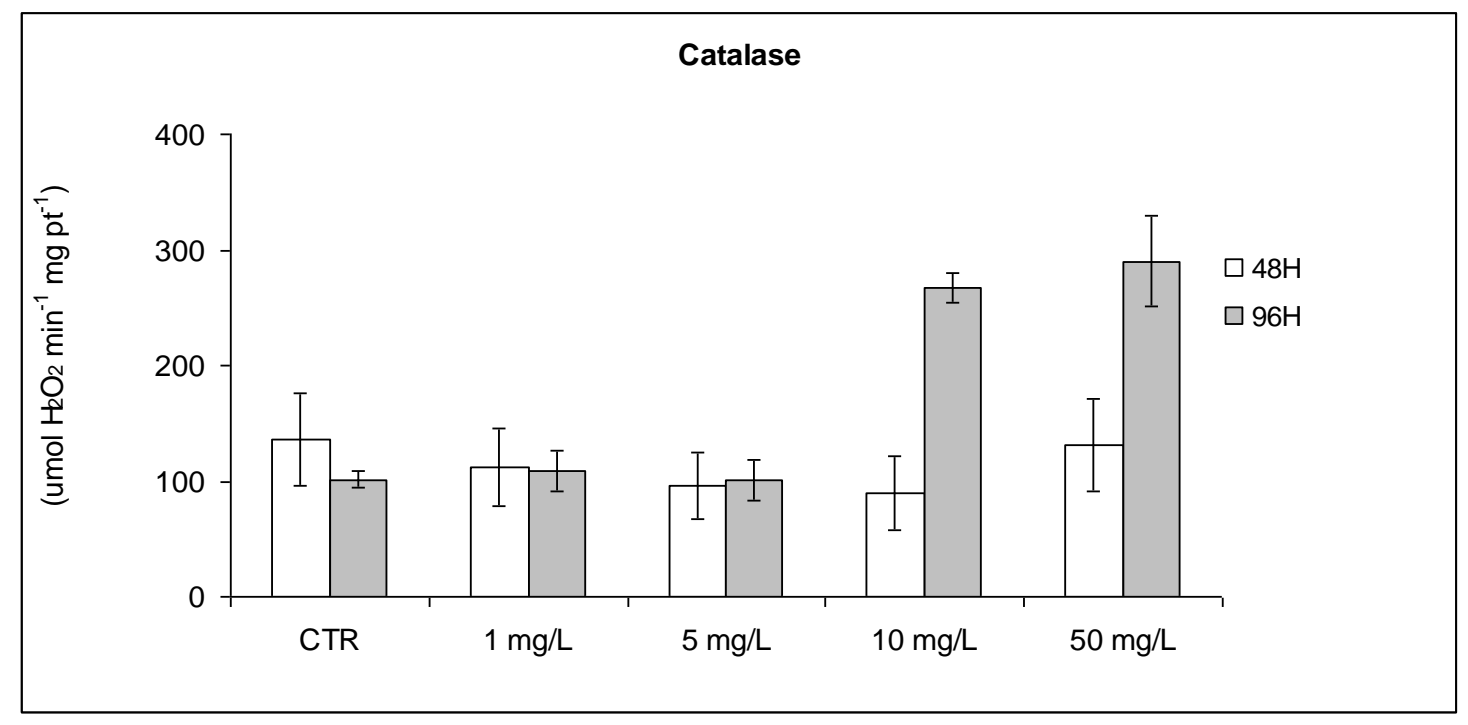

Fonte: Autores.

Em nosso trabalho, comparando-se o tempo de exposição, nas concentrações de 10 e $50 \mathrm{mg} \cdot \mathrm{L}^{-1}$ de $\mathrm{TiO}_{2}$ a atividade da CAT foi significativamente maior $(\mathrm{t}=-6,35$ e $\mathrm{p}=0,00 ; \mathrm{t}=-3,10 \mathrm{e} \mathrm{p}=0,005)$ nos animais expostos durante $96 \mathrm{~h}$ a nanopartícula. Entretanto, esses valores não diferiram significativamente entre si (Fig. 1).

Assim, podemos inferir que a exposição mais curta $(48$ h) não foi capaz de induzir a atividade da CAT nas $O$. niloticus testadas, mesmo quando expostas as maiores concentrações. Resultado semelhante foi descrito por Hao et al. (2009), que só observaram o aumento na atividade da CAT hepática de carpas (C. carpio) expostas a 10 e $50 \mathrm{mg} . \mathrm{L}^{-1}$ da NP-TiO $\mathrm{N}_{2}$ após

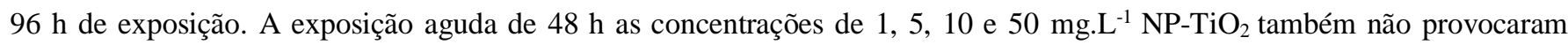
variação significativa na atividade da CAT hepática de peixes da espécie Prochilodus lineatus (Carmo et al., 2019). Dessa forma, curtos períodos de exposição a $\mathrm{NP}-\mathrm{TiO}_{2}$, mesmo em concentrações mais altas, parecem não interferir na atividade da CAT hepática.

Além do tempo de exposição, a concentração da NP é um fator importante e interferente na atividade das enzimas. Xiong, Fang, Yu, Sima \& Zhu (2011) não observaram variação na atividade da CAT hepática de zebrafish (Danio rerio) expostos a $5 \mathrm{mg} \cdot \mathrm{L}^{-1} \mathrm{NP}-\mathrm{TiO}_{2}$ e sugerem que a concentração testada, mesmo durante $96 \mathrm{~h}$, foi baixa para gerar EROs suficientes e ativar o sistema de defesa antioxidante. O mesmo pode ter ocorrido nas tilápias testadas em nosso trabalho, visto que, não observamos variação significativa na atividade da CAT hepática dos animais expostos as concentrações de $1 \mathrm{e} 5 \mathrm{mg} . \mathrm{L}^{-1} \mathrm{de} \mathrm{NP}$, 
mesmo durante $96 \mathrm{~h}$. Diferente de nossos resultados, Varela-Valencia et al. (2014) evidenciaram que mesmo em baixas concentrações $\left(0,1 \mathrm{mg} \cdot \mathrm{L}^{-1}\right.$ de NP-TiO${ }_{2}$ via intraperitonial), ocorre aumento nos níveis de transcrição do RNAm de CAT hepática de $O$. niloticus. Porém, cabe salientar que a via de administração foi diferente da testada em nosso trabalho. Na água as $\mathrm{NPs}-\mathrm{TiO}_{2}$ podem sofrer agregação, que consequentemente leva à sua precipitação e reduz a disponibilidade das NPs. Carmo

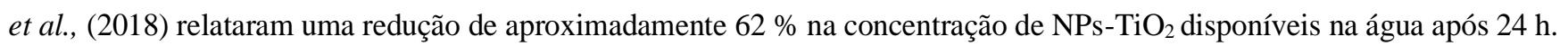

A principal função da CAT é diminuir a toxicidade do peróxido de hidrogênio $\left(\mathrm{H}_{2} \mathrm{O}_{2}\right)$ através da quebra dessa molécula em água e oxigênio. Assim, exposição a altas concentrações de $\mathrm{NP}^{-\mathrm{TiO}_{2}}$ podem estar relacionadas com a menor atividade da CAT observada nas tilápias, mesmo em períodos de exposição mais curto. Karthigarani e Navaraj (2012) observaram menor atividade da CAT em tilápias (O. Mossambicus) expostas a 50, 75 e 100 mg.L-1 de $\mathrm{TiO}_{2}$ durante $48 \mathrm{~h}$. Segundo os autores, o $\mathrm{TiO}_{2}$ pode ter inibido a atividade da CAT hepática, devido a sua alta capacidade de acumulação no

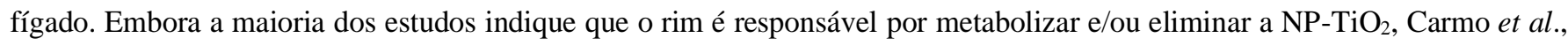
(2018) apontaram que o fígado também tem um papel de destaque nesses processos.

As $\mathrm{NP}_{-} \mathrm{TiO}_{2}$ tem uma característica específica de se ligar a enzima CAT por forças eletrostáticas e de ligações de hidrogênio, podendo, em altas concentrações inibir a atividade dessa enzima (Zhang, Cao, Tang \& Wang, 2014). A redução da atividade da CAT não foi observada em nosso estudo, porém, cabe ressaltar que as concentrações testadas em nosso trabalho foram menores que as testadas por Karthigarani e Navaraj (2012). A redução na atividade da CAT também foi observada em carpas (C. carpio) expostas a concentrações de $\mathrm{NP}^{-\mathrm{TiO}_{2}}$ de 2 a 4 vezes maiores que as testadas em nosso trabalho (Hao et al., 2009).

A presença de $\mathrm{NPs}_{-} \mathrm{TiO}_{2}$ também pode ter um papel importante na absorção de outros elementos, podendo influenciar na toxicidade de outros poluentes coexistentes no ambiente aquático. Zhang et al. (2007), observaram um aumento de 146\% da

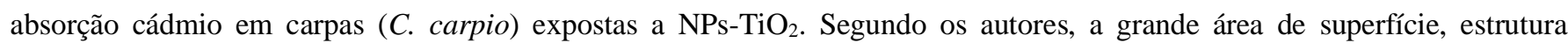
cristalina e reatividade de algumas nanopartículas pode facilitar o transporte de substâncias tóxicas poluentes no meio ambiente. Assim, as NPs podem atuar como transportador desses metais que podem se acumular no animal. Zhang et al. (2018) observaram que em sistemas aquáticos naturais a alta concentração de $\mathrm{NP}^{-\mathrm{TiO}_{2}}$ pode aumentar a toxicidade de outras nanopartículas, como a NP de prata. Em duas espécies de peixes (C. auratus e C. carpio), os metais cromo, ferro e níquel enriquecidos com $\mathrm{TiO}_{2}$ também foram responsáveis por lesões histopatológicas, alterações comportamentais e estresse oxidativo, caracterizado por alterações significativas nas atividades da CAT (Pirsaheb et al., 2019).

Diferentemente da CAT, já nas primeiras 48 h de exposição a $\mathrm{NP}_{-} \mathrm{TiO}_{2}$ nós observamos uma variação significativa na atividade da GST hepática dos peixes expostos a nanopartícula (Figura 2). 
Figura 2. Atividade da enzima hepática glutationa S-transferase (GST) de tilápia (O. Niloticus) expostas a 0 (controle), 1, 5 , 10 e $50 \mathrm{mg} . \mathrm{L}^{-1}$ da nanopartícula de $\mathrm{TiO}_{2}$ após 48 e 96 horas de exposição. Valores apresentados como média e desvio padrão. Letras iguais indicam valores sem diferença significativa $(p>0,05)$ e letras diferentes indicam valores estatisticamente diferentes $(\mathrm{p}<0,05)$. Letras maiúsculas referente a diferença entre as concentrações, letras minúsculas diferença referente ao tempo de exposição dentro do mesmo grupo. $\left(^{*}\right)$ indica diferença significativa entre o grupo controle.

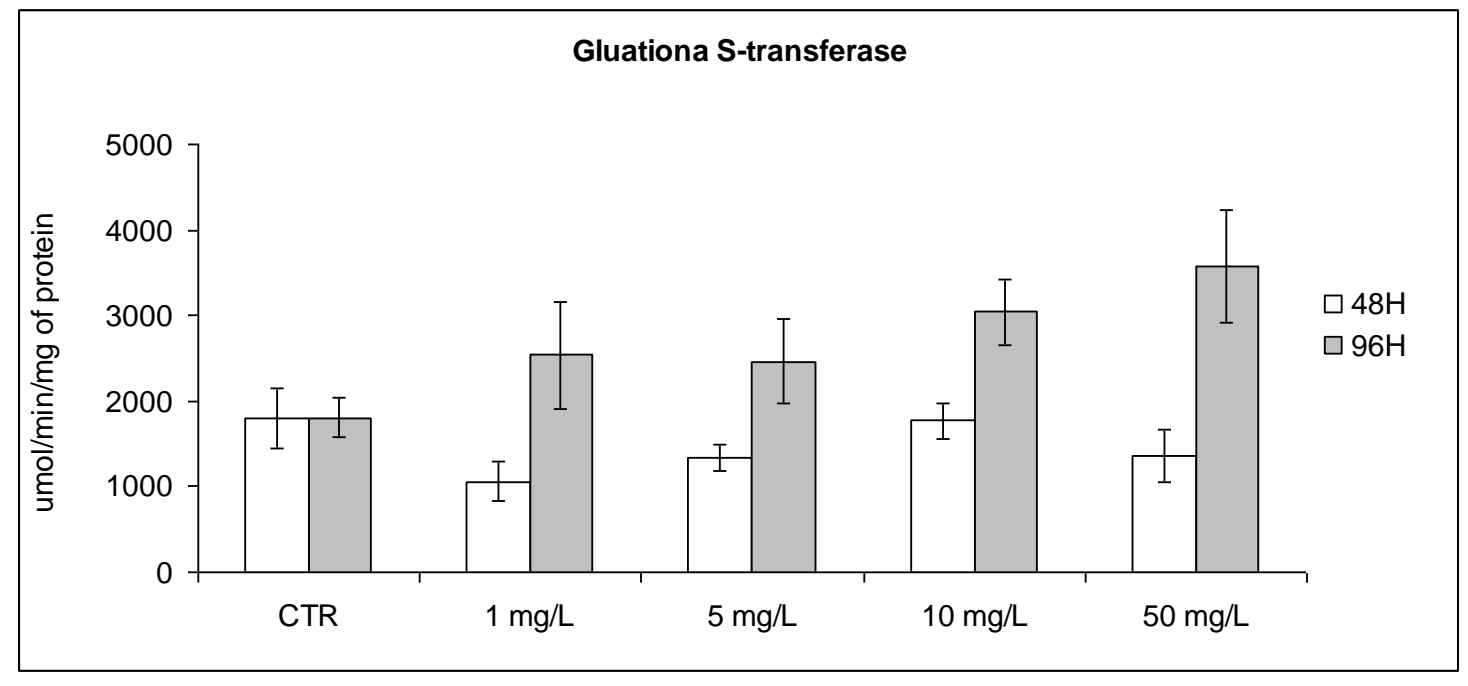

Fonte: Autores.

A atividade da GST foi significativamente menor $\left(\mathrm{F}=10,29\right.$ e p=0,00) nos animais expostos a 1,5 e $50 \mathrm{mg} \cdot \mathrm{L}^{-1} \mathrm{de} \mathrm{TiO}_{2}$, quando comparado com o grupo CTR. Entretanto, a atividade nos animais expostos a $10 \mathrm{mg} \cdot \mathrm{L}^{-1} \mathrm{de}^{\mathrm{TiO}}$ não variou em relação ao controle. A variação na atividade da GST observada nas tilápias logo nas primeiras 48h de exposição, pode estar relacionada a rápida ativação dessa enzima. Segundo Varela-Valencia et al. (2014), o pico da expressão gênica da GST hepática em $O$. niloticus ocorre nas primeiras $6 \mathrm{~h}$ de exposição, com subsequente redução na atividade até as $24 \mathrm{~h}$. Como em nosso trabalho a atividade da GST hepática foi determinada após $48 \mathrm{~h}$ de exposição, provavelmente esse comportamento inicial na ativação da GST não foi detectado.

A enzima GST também apresenta importância no processo de defesa do estresse oxidativo, visto que, protegem proteínas, lipídios e o DNA da oxidação (Benavides et al., 2016). A GST é uma importante enzima de biotransformação de fase II, e constitui a segunda linha de defesa contra a toxicidade causada pelos oxidantes. Ela atua conjugando xenobióticos, incluindo os aldeídicos produzidos durante a lipoperoxidação, com a glutationa na forma reduzida (GSH) tornando-os mais polares e facilitando a remoção intracelular (Canli, Dogan \& Canli, 2018). Assim, a GST hepática é conhecida por seu importante papel na defesa do organismo contra danos oxidativos nas células.

$\mathrm{Na}$ exposição de $96 \mathrm{~h}$, a atividade da GST variou significativamente somente nos animais expostos a $50 \mathrm{mg} \cdot \mathrm{L}^{-1}$ de TiO (Figura 2), sendo esta, maior quando comparada com as demais concentrações e seu grupo CTR ( $F=12,38$ e $p=0,00$ ). Nas demais concentrações testadas, nós não observamos diferenças significativas entre os tratamentos.

Segundo Bobori et al. (2020), a presença da $\mathrm{NP}_{-} \mathrm{TiO}_{2}$ pode ativar um mecanismo comum que envolve a estimulação do sistema imunológico com a produção de EROs, danos na membrana lisossomal, carbonilação de proteínas, peroxidação lipídica, danos ao DNA e apoptose. O sistema antioxidante é capaz de neutralizar o $\mathrm{H}_{2} \mathrm{O}_{2}$ e outras EROs formadas pelas NPs e, consequentemente, proteger o organismo do estresse oxidativo.

Na comparação entre o tempo de exposição, observamos que a atividade da GST hepática foi significativamente maior nos animais expostos durante $96 \mathrm{~h}$ a NP-TiO 2 , quando comparados com os valores obtidos nos animais expostos as mesmas concentrações durante o período de 48 h (Figura 2). Cabe salientar que, a atividade das enzimas antioxidantes, como a 
GST, pode aumentar ou ser inibida sob estresse químico, indicando que não há uma regra geral para a atividade dessas enzimas (Cheng, Zheng, Li, Richardson \& Lam, 2001; Hou et al., 2019).

A redução da atividade da GST também foi observada no tecido muscular de O. niloticus em expostas a 2 mg.L.-1 de NP-ZnO durante 7 dias, sendo essa redução mais acentuada após 14 dias de exposição (Canli et al., 2018). Entretanto, as mesmas concentrações testadas em nosso trabalho não provocaram alteração na atividade da GST de curimbas ( $P$. lineatus) expostos a $48 \mathrm{~h}$ a NP-TiO 2 (Carmo et al., 2019).

O tempo prolongado de exposição a altas concentrações parece ter um resultado diferente. A maior atividade da GST observada nas tilápias expostas a $50 \mathrm{mg} . \mathrm{L}^{-1}$ de $\mathrm{NP}-\mathrm{TiO}_{2}$ pode estar relacionado a um mecanismo compensatório ao estresse oxidativo, devido ao aumento em sua síntese, ao longo da exposição a NP (Abdelazim, Saadeldin, Swelum, Afifi \& Alkaladi, 2018). Carmo et al. (2018) também observaram maior atividade da GST hepática em P. lineatus expostos a 10 e $50 \mathrm{mg} . \mathrm{L}^{-1}$ de NP-TiO ${ }_{2}$ durante 14 dias. Sabe-se que a síntese de GST pode aumentar devido a um mecanismo adaptativo ao estresse oxidativo, o que pode explicar nosso resultado (Zhang, Sun, Zhang, Niu, Chen \& Crittenden, 2007). A maior atividade da GST também foi observada em outras duas espécies de peixes, D. rerio e C. auratus, expostas a 10 e $100 \mathrm{mg} . \mathrm{L}^{-1}$ de NP-TiO ${ }_{2}$ durante 7 dias (Diniz et al., 2013). Entretanto, após 21 dias de exposição os autores observaram redução significativa na atividade da enzima para ambas as espécies. Resultado semelhante foi observado em carpas (C. auratus) expostas a $100 \mathrm{mg} . \mathrm{L}^{-1}$ da NP-ZnO (Benavides et al., 2016). A redução da atividade da GST pode estar relacionada com a diminuição da síntese de GST por estresse severo, devido à concentração dessas nanopartículas em tecidos alvo, como o fígado. O estresse oxidativo severo pode suprimir suas atividades devido a uma perda de mecanismos de resposta ao estresse induzido (Zhang et al., 2007), resultado geralmente observado em peixes expostos a altas concentrações de $\mathrm{TiO}_{2}$ ou durante longos períodos de exposição. A redução na atividade da GST não foi observado em nosso trabalho, provavelmente devido ao tempo de exposição (4 dias), indicando que, durante esse período, o fígado parace ser capaz de produzir a GST para combater as EROs.

A maior atividade da GST também foi observada em outros animais aquáticos expostos a $\mathrm{NP}^{-\mathrm{TiO}_{2}}$, como em moluscos bivalves Mytilus galloprovincialis (Canesi et al., 2010) e Mytilus coruscos (Huang et al., 2018), em microcrustáceos Daphnia magna (Kim, Klaine, Cho, Kim \& Kim, 2010) e em diferentes órgãos de mamíferos (Kang, Kim, Lee \& Chung, 2008). Isso pode indicar que a ativação do mecanismo de resposta antioxidante funcione de maneira semelhante para

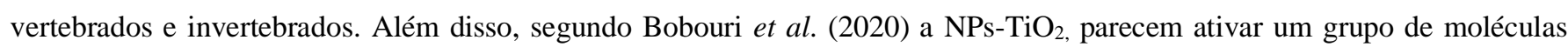
que são comum para animais aquáticos e terrestres, implicando a existência de um mecanismo conservado. Ainda em nosso trabalho, não observamos diferença significativa entre os resultados do grupo CTR (48 e 96 h) e não houve mortalidade dos animais durante os testes. Assim, podemos concluir que os peixes representam um modelo biológico relevante na área da nanoecotoxicologia, visto que, os resultados podem ser extrapolados para os mamíferos. Aparentemente, as nanopartículas de $\mathrm{TiO}_{2}$ causam efeitos semelhantes em peixes e ratos, sendo o fígado o tecido mais afetado (Zhang et al., 2018). Além disso, as respostas da atividade das enzimas CAT e GST se mostraram bons biomarcadores, podendo ser utilizadas para avaliação dos efeitos das NPs- $\mathrm{TiO}_{2}$ sobre os peixes.

\section{Conclusão}

Em nosso trabalho, a exposição de tilápias (O. niloticus) às concentrações de $\mathrm{NP}^{-} \mathrm{TiO}_{2}$ testadas foram capazes de provocar alterações significativas na atividade dos biomarcadores avaliados. Além da concentração, o tempo de exposição à nanopartícula parece influenciar na resposta das enzimas, aumentando sua atividade.

Esses resultados são importantes, pois, contribuem para o conhecimento do impacto final dos nanomateriais na saúde e no meio ambiente, bem como, sua interferência nos processos metabólicos dos animais. No entanto, são necessárias mais informações sobre os efeitos das $\mathrm{NP}-\mathrm{TiO}_{2}$ por períodos mais longos de exposição, se quisermos entender a vias bioquímicas 
pelas quais as nanopartículas são metabolizadas. Dessa forma, estudos complementares com a avaliação da exposição crônica serão realizados com objetivo de elucidar os efeitos da exposição de peixes as NPs.

\section{Agradecimentos}

A Fundação de Amparo à Pesquisa do Estado da Bahia (FAPESB), pela concessão de bolsa de Iniciação a Pesquisa (IC-PIBIC) a discente Theila dos Santos Santana e a piscicultura Aqua Vale (Ituberá, BA), pelo fornecimento dos peixes que foram utilizados neste trabalho.

\section{Referências}

Aebi, H. (1984). Calalase in vitro. Methods in Enzymology, 105, 121-126. https://doi.org/10.1016/S0076-6879(84)05016-3

Abdelazim, A. M., Saadeldin, I. M., Swelum, A. A., Afifi, M. M. \& Alkaladi A. (2018). Oxidative stress in the muscles of the fish Nile Tilapia caused by zinc oxide nanoparticles and its modulation by vitamins C and E. Oxidative Medicine Celluler Longevity, 1-9. https://doi.org/10.1155/2018/6926712

Benavides, M., Fernandez-Lodeiro, J., Coelho, P., Lodeiro, C. \& Diniz, M. S. (2016). Single and combined effects of aluminum (Al $\left.\mathrm{O}_{3}\right)$ and zinc (ZnO) oxide nanoparticles in a freshwater fish, Carassius auratus. Environmental Science and Pollution Research, 23(24), 24578-24591. DOI 10.1007/s11356-016-7915-3

Bobori, D., Dimitriadi, A., Karasialia, S., Tsoumaki-Tsouroufli, P., Mastora, M., Kastrinaki, G., Feidantsis, K., Alice Printzi, A., Koumoundouros, G. \& Kaloyiannic, M. (2020). Common mechanisms activated in the tissues of aquatic and terrestrial animal models after TiO ${ }_{2}$ nanoparticles exposure. Environment International, 138 (105611), 1-11. https://doi.org/10.1016/j.envint.2020.105611

Canesi, L., Fabbri, R., Gallo, G., Vallotto, D., Marcomini, A. \& Pojana, G., 2010. Biomarkers in Mytilus galloprovincialis exposed to suspensions of selected nanoparticles (Nano carbon black, C60 fullerene, Nano-TiO $\mathrm{T}_{2}$ Nano-SIO ${ }_{2}$ ). Aquatic Toxicology, 100(2), 168-177. doi: 10.1016/j.aquatox.2010.04.009

Canli, E. G., Dogan, A. \& Canli, M. (2018). Serum biomarker levels alter following nanoparticle $\left(\mathrm{Al}_{2} \mathrm{O}_{3}, \mathrm{CuO}, \mathrm{TiO}_{2}\right)$ exposures in freshwater fish (Oreochromis niloticus). Environmental Toxicology and Pharmacology, 62, 181-187. https://doi.org/10.1016/j.etap.2018.07.009

Carmo, T. L. L., Azevedo, V. C., Siqueira, P. R., Galvão, T. D., Santos, F. A., Martinez, C. B. R., Appoloni, C. R. \& Fernandes, M. N. (2018). Reactive oxygen species and other biochemical and morphological biomarkers in the gills and kidneys of the Neotropical freshwater fish, Prochilodus lineatus, exposed to titanium dioxide $\left(\mathrm{TiO}_{2}\right)$ nanoparticles. Environmental Science and Pollution Research, 25, 22963-22976. https://doi.org/10.1007/s11356-018-2393-4

Carmo, T. L. L., Siqueira, P. R., Azevedo, V. C., Tavares, D., Pesenti, E. C., Cestari, M. M., Martinez, C. B. R \& Fernandes, M. N. (2019). Overview of the toxic effects of titanium dioxide nanoparticles in blood, liver, muscles, and brain of a Neotropical detritivorous fish. Environmental Toxicology, 34(4), 457468. DOI: $10.1002 /$ tox.22699

Cheung, C. C. C., Zheng, G. J., Li, A. M. Y., Richardson, B. J. \& Lam, P. K. S. (2001). Relationship between tissue concentrations of polycyclic aromatic hydrocarbons and antioxidative responses of marine mussels, Perna viridis. Aquatic Toxicology, 52: 189-203. https://doi.org/10.1016/S0166-445X(00)00145-4

Clemente, Z., Castro, V. L., Feitosa, L. O., Jonsson, C. M., Maia, A. H. N. \& Fraceto, L. F. (2013). Fish exposure to nano-TiO 2 under different experimental conditions: Methodological aspects for nanoecotoxicology investigations. Science of the Total Environmental, 463-464, 647-656. http://dx.doi.org/10.1016/j.scitotenv.2013.06.022

Diniz, M. S., Matos, A. A., Lourenço, J., Castro, L., Peres, I., Mendonça, E. \& Picado A. (2013). Histological and biochemical effects of exposure to TiO 2 nanoparticles in livers of two freshwater fish species: Carassius auratus and Danio rerio. Microscopy and Microanalysis, $1-10$. doi: $10.1017 /$ S 1431927613013238

Hajirezaee, S., Mohammadi, G., Naserabad, S. S. (2020). The protective effects of vitamin C on mon carp (Cyprinus carpio) exposed to titanium oxide nanoparticles ( $\mathrm{TiO}_{2}$-NPs). Aquaculture, 518(734743), 1-12. https://doi.org/10.1016/j.aquaculture.2019.734734

Hao, L., Wang, Z. \& Xing, B. (2009). Effect of sub-acute exposure to $\mathrm{TiO}_{2}$ nanoparticles on oxidative stress and histopathological changes in Juvenile Carp (Cyprinus carpio). Journal of Environmental Science, 21, 1459-1466. DOI: 10.1016/S1001-0742(08)62440-7

Hou, J., Wang. L., Wang, C., Zhang, S., Liu, H., Li. S. \& Wang, X. (2019). Toxicity and mechanisms of action of titanium dioxide nanoparticles in living organisms. Journal of Environmental Science, 75, 40-53. https://doi.org/10.1016/j.jes.2018.06.010

Huang, X., Liu, Z., Xie, Z., Dupont, S., Huang, W., Wu, F., Kong, H., Liu, L., Sui, Y., Lin, D., Lu, W., Hu, M. \& Wang. Y. (2018). Oxidative stress induced by titanium dioxide nanoparticles increases under seawater acidification in the thick shell mussel Mytilus coruscus. Marine Environmetal Research, 137, 4959. https://doi.org/10.1016/j.marenvres.2018.02.029

Kang, S. J., Kim, B. M., Lee, Y. J. \& Chung, H. W. (2008). Titanium dioxide nanoparticles trigger p53-mediated damage response in peripheral blood lymphocytes. Environmental and Molecular Mutagenesis, 49, 399-405. https://doi.org/10.1002/em.20399

Karthigarani, M. \& Navaraj, D.P.S. (2012). Impact of nanoparticle on enzymes activity in Oreochromis mossambicus. Internation Journal of Scientific \& Technology, 1(10), 13-17.

Keen, J. H., Habig, W. H. \& Jakoby, W. B. (1976). Mechanism for the several activities of the glutathione S-transferases. Journal of Biological Chemistry, 251(20), 6183-6188. 
Research, Society and Development, v. 10, n. 5, e46810512829, 2021

(CC BY 4.0) | ISSN 2525-3409 | DOI: http://dx.doi.org/10.33448/rsd-v10i5.12829

Keller, A. A., McFerran, S., Lazareva, A. \& Suh, S. (2013). Global life cycle releases of engineered nanomaterials. Journal of Nanoparticules Research, 15(1692). doi:10.1007/s11051-013-1692-4

Kim, K. T., Klaine, S. J., Cho, J., Kim, S. H. \& Kim, S. D. (2010). Oxidative stress responses of Daphnia magna exposed to $\mathrm{TiO}_{2}$ nanoparticles according to size fraction. Science of the Total Environmental, 408(10), 2268-2272. doi:10.1016/j.scitotenv.2010.01.041

Mahaling, B. \& Dinabandhu, A. (2018). Nanotoxicology: the emerging nanoresearch. Sciencia Horticulture, 3(7), 14-16.

Martirosyan, A. \& Schneider, Y. J. (2014). Engineered nanomaterials in food: Implications for food safety and consumer health. International Journal of Environmental Research and Public Health, 11(6), 5720-5750. doi:10.3390/ijerph110605720

Paital, B. (2018). Removing small non-enzymatic molecules for biochemical assay of redox regulatory enzymes; An exemplary comments on "Antioxidant responses in gills and digestive gland of oyster Crassostrea madrasensis (Preston) under lead exposure. Ecotoxicology and Environmental Safety, 154, 337340. https://doi.org/10.1016/j.ecoenv.2018.01.051

Pham, C. H., Yi, J. \& Gu, M. B. (2012). Biomarker gene response in male Medaka (Oryzias latipes) chronically exposed to silver nanoparticle. Ecotoxicology and Environmental Safety, 78: 239-245. doi:10.1016/j.ecoenv.2011.11.034

Pirsaheb, M., Azadi, N. A., Miglietta, M. L., Sayadi, M. H., Blahova, J., Fathi, M. \& Mansouri, B. (2019). Toxicological effects of transition metal-doped titanium dioxide nanoparticles on goldfish (Carassius auratus) and common carp (Cyprinus carpio). Chemosphere; 215: 904-915. https://doi.org/10.1016/j.chemosphere.2018.10.111

Reeves, J. F., Davies, S. J., Dodd, N. J. F. \& Jha, A. N. (2008). Hydroxyl radicals (OH) are associated with titanium dioxide $\left(\mathrm{TiO}_{2}\right)$ nanoparticle induced cytotoxicity and oxidative DNA damage in fish cells. Mutation Research/Fundamental and molecular mechanisms of mutagenesis; 640: 113-122. doi:10.1016/j.mrfmmm.2007.12.010

Valavanidis, A., Vlahogianni, T., Dassenakis, M. \& Scoullos, M. (2006). Molecular biomarkers of oxidative stress in aquatic organisms in relation to toxic environmental pollutants. Ecotoxicology and Environmental Safety, 64, 178-189. doi:10.1016/j.ecoenv.2005.03.013

Varela-Valencia, R., Gómez-Ortiz, N., Oskam, G., Coss, R., Rubio-Piña, J., Río-García, M., Albores-Medina, A. \& Zapata-Perez, O. (2014). The effect of titanium dioxide nanoparticles on antioxidant gene expression in tilapia (Oreochromis niloticus). Journal of Nanoparticules Research, 16 (2369), 1-12. doi: $10.1007 / \mathrm{s} 11051-014-2369-3$

Xiong, D., Fang, T., Yu, L., Sima, X. \& Zhu, W. (2011). Effects of nano-scale $\mathrm{TiO}_{2}, \mathrm{ZnO}$ and their bulk counterparts on zebrafish: acute toxicity, oxidative stress and oxidative damage. Science of the Total Environmental, 409: 1444-1452. doi:10.1016/j.scitotenv.2011.01.015

Zhang, X., Sun, H., Zhang, Z., Niu, Q., Chen, Y. \& Crittenden, J. C. (2007). Enhanced bioaccumulation of cadmium in carp in the presence of titanium dioxide nanoparticles. Chemosphere, 67(1), 160-166. https://doi.org/10.1016/j.chemosphere.2006.09.003

Zhang, Y., Qiang, L., Yuan, Y., Wu, W., Sun, B. \& Zhu, L. (2018). Impacts of titanium dioxide nanoparticles on transformation of silver nanoparticles in aquatic environments. Environmental Science Nano, 5(5): 1191-1199. https://doi.org/10.1039/C8EN00044A

Zhang, H.M., Cao, J., Tang, B.P. \& Wang, Y.Q. (2014). Effect of $\mathrm{TiO}_{2}$ nanoparticles on the structure and activity of catalase. Chemico-Biological Interactions, 219, 168-174. http://dx.doi.org/10.1016/j.cbi.2014. 